\title{
Magnetic fields in the Pipe nebula region $\dagger$
}

\author{
Gabriel A. P. Franco ${ }^{1}$, Felipe O. Alves ${ }^{2}$ and J. M. Girart ${ }^{2}$ \\ ${ }^{1}$ Departamento de Física - ICEx - UFMG, Brazil \\ email: franco@fisica.ufmg.br \\ ${ }^{2}$ Institut de Ciències de l'Espai (CSCI-IEEC), Campus UAB-Facultat de Ciències, Torre \\ C5-Parell $2^{a}$, 08193 Bellaterra, Catalunya, Spain \\ email: [oliveira; girart]@ieec.uab.es
}

\begin{abstract}
Magnetic fields are supposed to play an important role in the formation and support of self-gravitating clouds and the formation and evolution of protostars in such clouds. We used $R$-band linear polarimetry collected for about 12000 stars in 46 fields with lines of sight toward the Pipe nebula to investigate the properties of the magnetic fields acting on this dark cloud complex.
\end{abstract}

Keywords. ISM: clouds - ISM: Pipe nebula - ISM: magnetic fields - techniques: polarimetric

\section{Introduction}

The Pipe nebula is a massive cloud $\left(10^{4} \mathrm{M} \odot\right.$, Lombardi, Alves \& Lada 2006) located at the solar vicinity (145 pc; Alves \& Franco 2007) which presents an apparently quiescent nature. The only known active star forming site, Barnard 59 (B 59), corresponds only to a small fraction of the entire cloud mass. The lack of star forming regions motivated us to investigate the magnetic properties that may be involved in the cloud evolution.

$R$ band linear polarimetry collected for about 12000 stars was used to investigate the properties of the polarization across the Pipe nebula dark cloud complex. Mean polarization vectors show that the magnetic field is locally perpendicular to the large filamentary structure of the Pipe nebula (the 'stem'), indicating that the global collapse may have been driven by ambipolar diffusion. The northwestern end of the nebula (B 59 region) is found to have a low degree of polarization and high dispersion in polarization position angle, while at the other extreme of the cloud (the 'bowl') we found mean degrees of polarization as high as $15 \%$ and a low dispersion in polarization position angle.

\section{Magnetic Fields}

The obtained dispersion in polarization angles were used to estimate the magnetic field strength for the observed areas by applying the modified Chandrasekhar-Fermi formula (Chandrasekhar \& Fermi 1953, Ostriker, Stone \& Gammie 2001). The volume density and line width of the molecular line emission associated with the dust that produces the observed optical polarization and extinction were estimated from the molecular data available in the literature. We found that the magnetic field strength in the B 59 region, stem, and bowl, in the plane of the sky, are about 17, 30, and $65 \mu \mathrm{G}$, respectively (Alves, Franco \& Girart 2008). The almost perpendicular alignment between the magnetic field and the main axis of the Pipe nebula's stem indicates clearly that this part of the cloud contracted in the direction of the field lines. Indeed, the magnetic pressure $\left(P_{\text {mag }}=\frac{B^{2}}{8 \pi}\right)$

$\dagger$ Based on observations collected at Observatório do Pico dos Dias, operated by Laboratório Nacional de Astrofísica (LNA/MCT, Brazil). 
of the diffuse part of the cloud (where is most of the mass) is the dominant source of pressure in the direction perpendicular to the field lines $\left(12 \times 10^{5}\right.$ and $2.6 \times 10^{5} \mathrm{~K} \mathrm{~cm}^{-3}$ for the bowl and stem, respectively), being higher than the pressure due to the weight of the cloud $\left(\mathrm{P}_{\text {cloud }} / \mathrm{k}=10^{5} \mathrm{~K} \mathrm{~cm}^{-3}\right.$, according to Lada, Muench, Rathborne et al. 2008).

\section{Conclusions}

- The global polarimetric properties of the Pipe nebula can be described as an increasing polarization degree along the filamentary structure from B 59 towards the bowl, while the dispersion in polarization angles decreases along this way.

- The results appear to indicate that there exist three regions in the Pipe nebula of distinct evolutionary stages:

(a) B 59, the only active star-forming site in the cloud. For the observed fields, we measure a large dispersion in polarization angle, low polarization degree, and a estimated magnetic field strength of $17 \mu \mathrm{G}$.

(b) The 'stem', which collapsed by means of ambipolar diffusion but has not yet given birth to stars. It appears to represent a transient evolutionary state between B 59 and the bowl. We estimated an average magnetic field strength of $30 \mu \mathrm{G}$ for this part of the cloud.

(c) The 'bowl', which shows the highest values of mean polarization and lowest values of dispersion in polarization angle. The dust grains in the bowl seems to be highly aligned by the rather strong magnetic fields that permeates this part of the cloud $(\mathrm{B} \approx 65 \mu \mathrm{G})$.

Further details will be published elsewhere (Franco, Alves \& Girart 2009).

\section{Acknowledgements}

We thank the staff of the Observatório do Pico dos Dias (LNA/MCT, Brazil) for their hospitality and invaluable help during our observing runs. Drs. A. M. Magalhães and A. Pereyra are acknowledged for providing the polarimetric unit and the software used for data reductions. This research has been partially supported by CEX APQ1130-5.01/07 (FAPEMIG, Brazil) and AYA2005-08523-C03 (Ministerio de Ciencia e Innovación, Spain).

\section{References}

Alves, F. O. \& Franco, G. A. P. 2007, A\&\&A 470, 597

Alves, F. O., Franco, G. A. P., \& Girart, J. M. 2008, A\&GA 486, L13

Chandrasekhar, S. \& Fermi, E. 1953, ApJ 118, 113

Franco, G. A. P., Alves, F. O., \& Girart, J. M. 2009, in preparation

Lada, C. J., Muench, A. A., \& Rathborne, J. et al. 2008, ApJ 672, 410

Lombardi, M., Alves, J., \& Lada, C. J. 2006, A\&A, 454, 781

Ostriker, E. C., Stone, J. M., \& Gammie, C. F. 2001, ApJ 546, 980 